\title{
Cigarette smoke factor aggravates pancreatitis
}

Aryl hydrocarbon receptor (AhR) ligands, which are components of cigarette smoke, exacerbate chronic pancreatitis in mice via the increased production of IL-22, according to new research.

Chronic pancreatitis is a progressive inflammatory condition involving the destruction of pancreatic tissue owing to fibrosis. Cigarette smoke is a known risk factor for chronic pancreatitis, but the mechanism underlying this association is unknown. AhR ligands have critical roles in immune regulation (such as in T-cell differentiation), and several AhR ligands occur in cigarette smoke.

Jing Xue and colleagues, therefore, investigated whether AhR ligands in cigarette smoke might promote chronic pancreatitis. The team administered 2,3,7,8-tetrachlorodibenzo-p-dioxin (TCDD), an AhR agonist and a component of cigarette smoke, to mice with chronic pancreatitis induced by caerulein injection. The mice showed histological damage, decreased pancreas size and increased expression of fibrosis-associated genes compared with mice with chronic pancreatitis that did not receive TCDD. However, TCDD did not induce pancreatitis in healthy control mice, suggesting that cigarette smoke alone is not sufficient to induce pancreatitis. The TCDD-treated mice with chronic pancreatitis were found to have increased levels of the cytokine IL-22, and an increased number of IL-22-expressing T cells, compared with mice with chronic pancreatitis that did not receive TCDD. Treatment with an IL-22-neutralizing antibody alleviated the effects of TCDD treatment, whereas administration of IL-22 worsened fibrosis in TCDD-treated mice, suggesting that IL-22 plays a crucial part in AhR-agonist-induced fibrogenesis. Importantly, the team also found higher serum IL-22 levels in humans who smoke than in non-smoking individuals or individuals who have stopped smoking for $>1$ year.

"IL-22 levels might help us in identifying those at risk of developing chronic pancreatitis," explains corresponding author Aida Habtezion, "We want to do more human studies to validate the data we presented. The implication is that we can block IL-22 signalling to decrease the progression of chronic pancreatitis."

\section{Charlotte Ridler}

ORIGINAL ARTICLE Xue, J. et al. Aryl hydrocarbon receptor ligands in cigarette smoke induce production of interleukin-22 to promote pancreatic fibrosis in models of chronic pancreatitis. Gastroenterology http://dx.doi.org/ 10.1053/j.gastro.2016.09.064 (2016) 